of its existence being discoverable by a reasonable exploration from the front. If the diagnosis were made the best course to pursue in treating such a case would be to resect a couple of ribs posteriorly, incise the diaphragm, and evacuate the pus through the pleura. This route should be chosen for two reasons-because it is the nearest by which it could be reached and also because, although the passage of this highly septic material through the pleura would not be free from risk, such risk would be very much less than conduction through the peritoneal cavity, the only other possible method of exit. If unrelieved by operation these abscesses prove very fatal from septicxmia and often infect the pleura and lung of the same side. They sometimes occur as the result of a minute perforation of the stomach which has been closed by adhesions to neighbouring organs and either has not been diagnosed or is accompanied by symptoms not severe enough to make the need for immediate operation clear. In these cases the few drops of stomach contents which escape are sufficient to start the septic process which results in the formation of an abscess in an inaccessible position which soon endangers life. This furnishes an additional reason for the need of imme diate operation if the diagnosis of perforation can be made. Such abscesses may occur between the diaphragm and the left lobe of the liver, between the stomach and the liver, between the stomach and the spleen, ${ }^{2}$ and lastly between the diaphragm and the right lobe of the liver. In this connexion it is well to remember that in a very large percentage of cases of perforating ulcer the lesion has been found on the anterior surface of the stomach.

Prevention of this unfortunate complication is better than cure, and sub-phrenic abscess can be largely guarded against by the technique observed during the performance of an operation for perforated gastric ulcer. The convex surface of the liver and the pouch that exists above its posterior attachment is the one spot in the abdomen which is likely to be overlooked in the cleansing process if the operator has not this region in mind, and it is a part to which the experience of these two cases has taught me especial attention should be given. In the second case after the toilet of the abdominal cavity had been completed I washed out several ounces of escaped stomach contents from above the liver, which would almost certainly have caused suppuration if left. In both cases the same plan of cleansing was followed, and the results obtained would induce me to repent this method. The lips of the wound being held apart and lifted up, large quantities of boiled water were poured into the abdomen out of jugs, the size of those used in bedrooms, until it returned clear. This is much preferable to the douche as the volume of water flushes out the cavity very effectually, and it also effects a considerable saving in time. Afterwards the remaining fluid was removed with sponges and wiping was resorted to in any suspicious situations. In cleansing the sub-phrenic space a rubber tube attached to a douche was pushed well to the back of the liver on either side of the suspensory ligament, and by raising the costal margins and depressing the liver, the whole of the convex surface was in this way cleansed. After the water returned clear a flat sponge was introduced down to its posterior attachments and the space wiped out. In the first case the region in question was not cleaned out so thoroughly as this; had this plan been adopted no doubt a successful result would have been obtained.

With respect to the incision it was the same in both cases, a median one, extending from just below the xiphoid cartilage to the umbilicus, or a little below it. This should be sufficient for the closure of a gastric ulcer wherever situated, without any side incisions or cross cuts. Through this opening the whole of the interior of the abdomen can be reached and the various localities where food is likely to collect-the loins, Douglas's pouch, \&c.-can be effectually washed and afterwards sponged out. I found no difficulty in passing the hand into the pelvis and wiping its floor with a sponge. In both cases the wound was completely closed and no drainage employed. In the second case the ulcer was excised. ${ }^{3}$ The specimen showed

${ }^{2}$ An interesting specimen of abscess in this situation was shown to the meeting by Mr. W. J. Stephens. The patient, a middle-aged woman, died from what was thought to be a malignant growth under the liver. She was only seen by Mr. Stephens shortly before death, and no clear history could be obtained. Post mortem an ulcer of the stomach was found which had leaked through a minute opening and occasioned an abscess containing nearly a pint of matter, lying in the left hypochondriac reglon bethy

3 Both the specimen and the patient were exhibited at the meeting. well its clean punched-out appearance on the mucous surface and the small round perforation through the peritoneal coat. The question as to whether excision is advisable is still an open one. Some writers deprecate it on account of (1) the additional time consumed; (2) the additional hæmorrhage occasioned; (3) the increase of shock to the patient by converting a small hole into a large gap, requiring a great number of sutures for its closure ; (4) the risk of a considerable addition to the escape of the gastric contents ; and (5) the difficulty in closure owing to protrusion of the mucous coat. These objections in many cases would be real enough to make the operator decide to give closure the preference over excision. In Case 2 the local appearances indicated clearly that the ulcer was a small and deep one, but even this left on its removal a wound in the stomach wall one and a half inches long by three-quarters of an inch broad, and required fourteen sutures for its closure. I have now the satisfaction, however, of knowing that the woman's disease is cured, that the previously existing symptoms have disappeared, that her digestion is normal, and that she has, I believe, been completely restored to health.

Nottingham.

\section{A CASE OF}

\section{STRANGULATION OF THE ILEUM BY PASSAGE THROUGH A CONGENITAL MESENTERIC HOLE; OPERATION; RECOVERY.}

\section{BY J. STANLEY KELLETT SMITH, F.R.C.S. ENG.}

A GIRL, aged fifteen years, was seized, while walking in the street on the afternoon of June 23rd, 1897, with violent abdominal pains, quickly followed by persistent vomiting. Mr. Bassett Jones, of Aberystwith, who saw the girl the next morning, reported on her condition then as follows: "The patient complained of severe pain referred to the umbilical region; the pain was continuous, but accompanied with paroxysmal exacerbations about every ten minutes, during which the patient suffered great agony. The tongue was furred. The pulse was 90 , sharp and small; the respirations were 20 ; and the temperature was $98.2^{\circ} \mathrm{F}$. The abdomen was flaccid and flat, with the walls moving on respiration; there was no local swelling to be seen. It was resonant on percussion, but not tympanitic; there was a small area of dulness in the right iliac fossa, over which there was some tenderness on pressure. Palpation revealed nothing, except a sense of resistance over the above-mentioned area of dulness. There was no passage of flatus or fæces. Vomiting was frequent and of a bilious charaiter ; the act was not preceded by much nausea and the stomach emptied itself without apparent effort. Rectal examination revealed only the presence of small scybala." The lower bowel was emptied by enemata and everything that could be suggested in the way of palliative treatment was tried, but without relief to the symptoms. I saw the patient in consultation on June 27th, four days after the onset of the illness. Intestinal obstruction had remained complete, and she was then in a state of great exhaustion. The pulse was 140 and of very unpromising character. The respirations were 32 . The abdomen was tympanitic, excepting a small patch of dulness in the right iliac region, and over this patch any pressure upon palpation was strongly resented by the muscles of the abdominal wall. When I saw her vomiting had just taken place, the ejected matter consisting of diluted bile with particles of curd of the milk which had been given in small quantity as food. There was no suspicion of fæcal odour. Operation was advised and immediately carried out. The abdomen was opened from the umbilicus to the pubes. Coils of distended and congested small intestine bulged through the wound and the escape of these was encouraged until the disposition of the mesentery could be comfortably made out. The gut was then carefully drawn from the abdominal cavity, proceeding always downwards, until the lower part of the ileum was reached, tender manipulation being here necessary owing to

A paper read before the Liverpool Medical Institution on Oct.21st 
the greatly increased congestion of the bowel. The cause of mischief was found in the shape of a hole in the lower part of the mesentery, pyriform in shape, measuring some two and a half inches by two inches, with its larger end close to the mesenteric attachment of a small loop of bowel, which, taking its upper and lower limits as fixed points, had swung itself through the hole and thus become strangulated. The parts were judged capable of recovery and so were restored to normal position, recurrence of the accident being guarded against by closure of the aperture. The exposed intestines were then washed with weak psrchloride solution and replaced in the abdominal carity with some difficulty, but without the necessity of puncture for gas drainage. The total time of operation occupied thirty-five minutes. Recovery was uninterrupted. Flatus passed on the day following the operation, and the bowels were voluntarily moved on the fourth and subsequent days.

Remarks,-Holes may occur in the mesentery as the result of disease, of injury, or of congenital malformation. The absence of any sign of previous disease or injury and the negative history there of, the smooth thickened margin of the aperture, its size and the regularity of its outline, and e:pecially its situation in the lower part of the mesentery, at the spot where such developmental deficiencies most frequently occur, lead me to regard this case as a typical example of a congenital mesenteric foramen.

Liverpool.

\section{ATONIC APHONIA}

BY ALEXANDER WALLACE, M.D. OXON. M.R.C.P. LOND.

SENIOR PHISICIAN TO THE ESSEX AND COLCHESTER HOSPITAL.

AFTER reading Dr. Bastian's clinical lecture on Hysterical Mutism and Other Functional Speech Defects, published in THe LANCET of Sept. 25th, 1897, p. 775, it struck me that the following case might be interesting as presenting some points of difference to the cases mentioned by him in his paper.

A man, azed thirty-seven years, married, the parent of two children, the youngest of whom was five years old, came to consult me on July 7th, 1897. Eighteen weeks before he had suddenly lost his speech, never having been dumb before. His hearing and other senses were perfect, and his general health, he said, was good, but he had never been strong. His parents had both died from old age. About a week after the seizare he, by the wish of his medical attendant, went to a London hospital, where, according to his account, he was an hour under examination. His larynx was examined externally and by the laryngoscope, and he was somewhat roughly treated, he said, to make him cry cut, but to no purpose, and he had remained in the same dumb state since. He was a rather spare, short man, of nervous habit, and somewhat depressed. His wife accompanied him. He wrote in my presence statements regarding himself coherently and accurately ; attempting to speak, the lips and tongue movements were correctly made as if speaking, but no sound issued, not even a whisper; this his wi e stated was his then normal condition. He complained of some languor and feebleness, with restlessness at night. There was no cough; his appetite was bad, but the bowels were regular and the urine was acid. His memory was good. Sometimes he felt a sudden fluttering at the præcordia with headache. Sensation was unimpaired. Locomotion was not so good as before. "He knows exactly what be wants to say, and frames the words with his lips, but no scun 1 comes." The features were straight and the tongue was not deflected; no trace of any prior cerebral attack could be detected. The pulse was irritable, 90, of good volume but rather tense. Examination of the neck and external larynx revealed nothing but some old superficial cicatrices. The heart sounds were normal, but rather weak; cardiac dulness was normal. The liver, spleen, and abdomen were normal. The battery had, he told me, been applied to him both at the hospital in London and afterwards by his medical attendant both externally to the larynx and internally, but without any result. The case seemed to me to be unusual because: (1) there was no evidence or history of any cerebral implication; (2) there was not the slightest whisper, such as we hear in hysterical mutism; (3) the mouth and lips were correctly acting as if articulating, yet no sound issued; and (4) his written answers and bis written sentences were correctly given and spelt. On further inquiry I elicited that twice since the attack during the first month he had pronounced the names of people distinctly and correctly, but felt weak and vertiginous afterwards, with pain at the precordia and great pallor; that he was a great walker, doing generally ten miles a day; that he was restless and excitable, brandishing his arms much; and that he smoked six pipes a day and indulged in coitus three times a week. I thought I saw daylight in these faulty habits. I cut down the coitus to once a fortnight, the pipes to two per day, and a bicycle was to be used in moderation instead of walking ten miles a day. I gave him an acid mixture with five minims of solution of strychnia and one and a half grains of quinine three times a day. To my surprise on July 16th the patient came to me to say that on the 14th he spoke audibly, "I feel better," and that he has since spoken a little each day in the evening; he spoke to me that day clearly; in a low, distinct voice he said that he slept befter. The pulse was 80 and soft. I saw him again on July 29rth; he had quite recovered and had had no return of the mutism. It seems to me that "atonic mutism" is the right description of what I suppose is a somewhat unusual bat happily an easily recoverable form. 1 bave recently heard that he remains quite well.

Colchester.

\section{otrinital athotes:}

\section{MEDICAL, SURGICAL, OBSTETRICAL, AND} THERAPEUTICAL.

\section{PERMANGANATE OF POTASSIUM AND OPIUM} POISONING.

By ReginaLd NivoH-SMrth, M.R C.S. RiNG., L.R.C.P. LOND.

LATE SENIOR HOCSE PHYSICIAN AND CHLOROFORMIST, WESTHYNSTER HosPTix.

THE following case of accidental opium poisoning with successful treatment occurred whilst I was in charge of the casualty department of Westminster Hospital.

A male adult, aged thirty-five years, well nourished, gave on his recovery the following history: "At four o'clock this morning I took two tablespoonfuls of laudanum in mistake for black draught, after which I went to bed, and on being called at seven I thought something must be wrong as I felt so drowsy. I examined the bottle and found I had made a mistake. I then took the proper black draught, got up, and went for a walk. On passing the hospital I felt so very sleepy that $I$ thought it best to come in for treatment." When I saw him, four hours after he had taken the opium. the patient was asleep on one of the seats in the surgery. I immediately roused him, gave him some strong coffee and a bypodermic injection of caffeine, three grains. Two porters were instructed to walk the patient about. His pupils at the time were contracted and failed to respond to light; the mouth was dry ard the tongue coated; the skin was cold and clammy. The pulse was 108 and feeble, respiration was somewhat laboured, and the muscles were relaxed. He could only just be roused by loud shouting. Two more caffeine injections were administered with no beneficial result. I now decided to try permanganate of potash, which I gave in the form of the liquor, four drachms. As a result there was slight improvement. Soon after I again gave him four drachms, thus making in all one ounce, $=4.8 \mathrm{gr}$. Fifteen minutes later the patient romited a large quantity of black fluid. From then his recovery was rapid and complete. He was, as is usual in these cases, admitted into one of the medical wards and detained for forty eight hours, $m y$ instructions to the nurse being to watch the patient closely and to administer nourishment every three hours. On testing the urine it was found to be normal. There was no meconic acid. It will be seen that the draught taken by mistake contained $33 \mathrm{grs}$. of opium or 3.3 grs. of morphine.

Dr. William Moor, of New York, ascertained that one grain of permanganate of potash oxidises one grain of morphine. With such undisputed evidence before us it is surprising that such a reliable and easily attainable antidote 